\title{
Estrous activity in the white rat: A function of time spent in activity'
}

EPHRAIM PERETZ ${ }^{2}$ AND JAMES R. WHITMAN

VETERANS ADMINISTRATION HOSPITAL, AMERICAN LAKE. WASHINGTON

The estrous activity cycle of the rat was analyzed to determine whether the variation in activity is accounted for by changes in rate of responding or duration of daily periods of activity. Running-wheel data indicated that time in activity is the critical variable.

The sexually mature female rat exhibits an estrous activity cycle (Hemmingsen \& Krarup, 1937; Slonaker, 1924; Wang, 1923) defined by the regular recurrence of changes in amount of activity, with a peak in total daily activity every four or five days. As behavior associated with the estrous cycle, these changes differ from sexual receptivity in that they are modifications of a type of behavior present during the anestrous as well as the estrous phases of the cycle. The amount of activity can be thought of as the product of the amount of time the animal is active and the rate of activity. The purpose of the study reported here was to determine the relative contribution of these two factors to variation in activity over the course of the cycle.

Nine albino female rats bred in the laboratory and 128 days old at the start of the experiment were studied for a continuous period of 105 days. The animals were maintained in Wahmann activity wheels housed in a light-proofed and air-conditioned room. The room temperature was kept at $70-75^{\circ} \mathrm{F}$, and fluorescent overhead lighting was provided daily for a $12 \mathrm{~h}$ period starting at 8:00 AM. Food and water were always available. Recording began after an adaptation period of 25 days.

Activity was measured in terms of number of revolutions of the wheel. At each revolution, a cam on the wheel operated two circuits by means of a microswitch attached to the wheel frame. One circuit was connected to an impulse counter that was photographed hourly. A spring-wound and an electric clock provided checks on the timing of the photographs. The other circuit was connected to an Esterline-Angus event recorder, the paper speed of which was fast enough to record separately every revolution. The counters and the event recorder were housed in a room separate from the experimental room.

Four measures of activity were obtained: total daily revolutions (D Rev); total hourly revolutions (H Rev); total time occupied by bursts of activity (BT); and rate of activity in revolutions per min (IR). A burst of activity was defined as a series of consecutive revolutions lasting at least $30 \mathrm{sec}$ during which each revolution was completed in less than 5 sec. IR was determined by doubling the number of revolutions occurring during a $30 \mathrm{sec}$ period to produce a rate per min value and was determined for the first $30 \mathrm{sec}$ of the last burst in each hourly period.
D Rev, H Rev, BT, and IR were analyzed for three eight day periods covering the early, middle, and late periods of the experiment. Each period was selected so that Day 1 was the second day before a day of peak activity. The analysis was based on the data from three $\mathrm{Ss}^{3}$ two (S6 and S8) that had the most regular estrous activity cycles and one (S1) that had the least regular cycle, where the degree of regularity is measured by the proportion of cycles with the modal duration. The means of corresponding days across periods are plotted for each $\mathrm{S}$ in Fig. 1a. A four day estrous activity cycle is evident for all Ss. It can be seen that the curves for D Rev and BT parallel each other closely, whereas there is no apparent relationship between $D$ Rev and IR. The figure also shows that IR varied little from day to day, ranging between 40 and 50 revolutions per min (IRs of zero were not included in the analysis), and was approximately the same for all three animals. It is also interesting that IR was the least variable for the $S$ with the most regular cycles (S8), and most variable for the $S$ (S1) with the least regular cycles. Thus, for D Rev over the course of the estrous activity cycle, the critical factor is the total time spent in activity.

Hourly values were then analyzed to determine the correlation between the measures $\mathrm{H}$ Rev, BT, and IR for the three Ss. The correlation coefficients (Pearson's $r$ ) for the three Ss were: for $H$ Rev and BT, .98, .99 , and .99; for $H$ Rev and IR, .42, .43, and .52; and for $\mathrm{BT}$ and IR, $.41, .40$, and .54 . The relative magnitude of these values is consistent with the conclusion based on the daily averages. The relationship between $H$ Rev

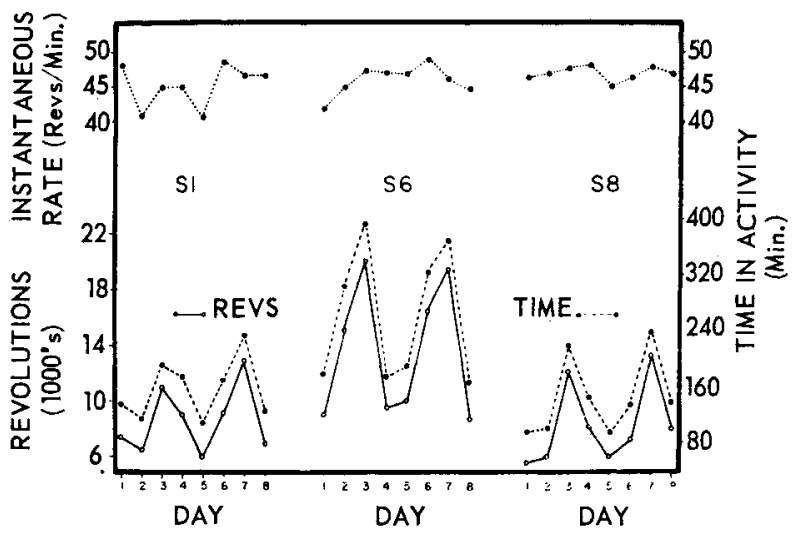

Fig. 1a. Daily mean values of the measures used in the analysis of daily data for three Ss. The three lines are the values for (from top to bottom) instantaneous rate (IR); time in activity (BT); and total number of revolutions (D Rev). Each point is the mean for three days. 


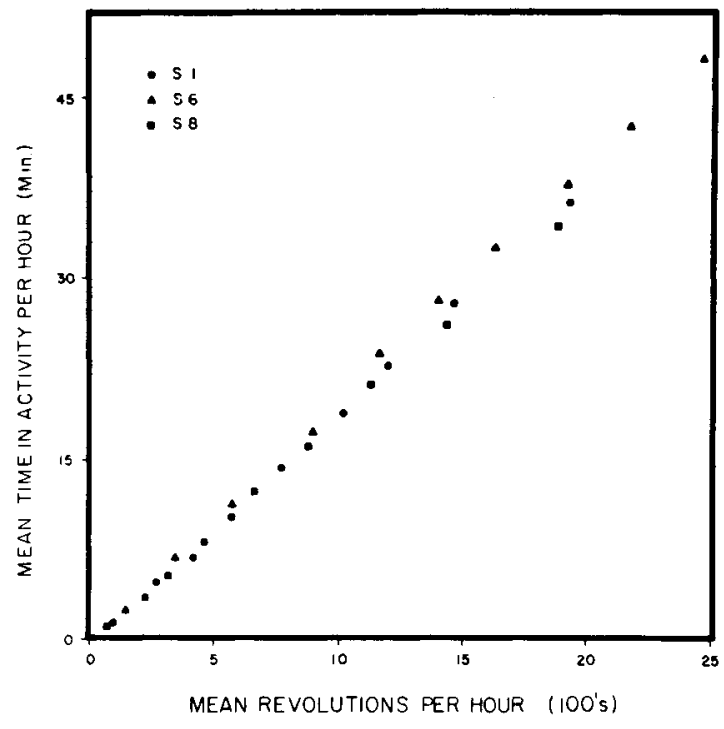

Fig. 1b. Mean time in activity (BT) as related to mean number of revolutions per hour (H Rev), for three Ss. Each point is the average of 25 observations.

and hourly BT is shown in Fig. 1b. In Fig. 1b, H Rev were ranked in order of magnitude and grouped in units of 25 values each. The mean BT for each group of 25 values is shown plotted against the mean $H$ Rev for the group. The linear relationship between these two measures is clear. It is also apparent that the slopes are nearly equal for all three Ss.

To determine whether or not this relationship held when different criteria were used for BT, a burst was defined as a period of activity of at least 3 min duration (4 Ss) and again as a period of activity of at least $6 \mathrm{~min}$ duration ( $5 \mathrm{Ss})$. For this analysis D Rev and daily BT were measured for all nine Ss over 13 consecutive days that followed the last eight day period in the first analysis. The correlations between BT thus defined and D Rev were comparable to those for hourly data. Pear- son's $\mathrm{r}$ values ranged between .95 and .99 , with a mean of .98; the conclusion is that the relationship between $\mathrm{BT}$ and $\mathrm{H}$ Rev was not due to the specific definition of BT used for the analysis.

The amount of activity of the normal adult female rat, as measured in the activity wheel, is determined almost entirely by the duration of periods of activity. Since the relationship is not specific to the estrous cycle, it may also be independent of age and sex. It is not clear why the number of revolutions is so closely paralleled by the duration of activity. Stellar \& Hill (1952) found that when rats vary the amount of water intake, they do so by varying the amount of time spent in drinking; the rate of lapping is constant. The present study indicates that there is a similar relationship between the amount of activity emitted and the rate at which the activity occurs for the female rat.

\section{References}

Hemmingsen, A. M., \& Krarup, N. B. Rhythmic diurnal variations in the oestrous phenomena of the rat and their susceptibility to light and dark. Kgl. Danske Vidensk. Selsk. Biol. Med., 1937, $13,1-61$.

Slonaker, J. R. The effects of pubescence, oestration, and menopause on the voluntary activity of the albino rat. Amer. J. Physiol., 1924, 68, 294-315.

Stellar, E., \& Hill, J. H. The rat's rate of drinking as a function of water deprivation. J. comp. physiol. Psychol., 1952, 45, 96102.

Wang, G. H. Relation between "spontaneous" activity and oestrous cycle in the white rat. Comp. Psychol. Monogr., 1923, 2, 1-27.

\section{Notes}

1. We thank Mrs. Hildegund Holloway and Mr. N. Roberts for assistance in collection and analysis of data, and Mrs. Mary J. Beaver for assistance in prtparation of records. We also acknowledge the assistance of the Western Research Support Center, VAH, Sepulveda, California for the computer processing of the data. 2. Present address: Oregon Regional Primate Research Center, $505 \mathrm{NW} 185$ th Ave., Beaverton, Oregon 97005.

3. For the three Ss, 99.4,99.3, and 98.6 percent of the activity occurred during periods for which BT could be measured (i.e., met the criteria for a "burst").

Comment on Clark and Bremner by Robert Boice and Carol Boice, The University of New Mexico

Clark \& Bremner (1967) state unequivocally that kangaroo rats do not drink water. Although there are many reports of Dipodymus living on air-dried food without drinking (Schmidt-Nielson, 1964), Richter (in Howell \& Gersh, 1935), among others, notes that kangaroo rats will consume up to $10 \mathrm{cc}$ of free water per day.

Ten Dipodymus ordii in our laboratory, maintained on Wayne Mouse Breeder Blox, have shown a mean intake of $8.8 \mathrm{cc}$ free water per day over as many as 143 days of ad lib availability and a mean intake of $3.9 \mathrm{cc}$ free water per day over as many as 70 days of $1 \mathrm{~h}$ per day availability of water. The behaviors of the kangaroo rats when on the $23 \mathrm{~h}$ deprivation schedule suggest great eagerness for the water bottle.

\section{References}

Clark, R. H., \& Bremner, F. J. Toward an electrophysiological identification of a primary drive factor. Psychon. Sci., 1967, 8, 113-114.

Howell, A. B., \& Gersh, 1. Conservation of water by the rodent Dipodymus. J. Mammal., 1935, 16, 1-9.

Schmidt-Nielsen, K. Desert animals. New York: Oxford University Press, 1964. 